\title{
LA-UR- $98-1162$
}

Approved for public release distribution is unlimited.

DESCRIPTION OF THE BDD-IIR: ELECTRON AND PROTON SENSORS ON THE GPS

T. E. Cayton, NIS-2

D. M. Drake, NIS-2

K. M. Spencer, NIS-4

M. Herrin, NIS-4

T. J. Wehner, NIS-4

R. C. Reedy, NIS-2

Submitted to:

For external distribution

DISTRIBUTION OF THIS DOCUMENT IS UALIMTED $q^{\prime}$

\section{Los Alamos}

NATIONAL LABORATORY

Los Alamos National Laboratory, an affirmative action/equal opportunity employer, is operated by the University of Califomia for the U.S. Department of Energy under contract W-7405-ENG-36. By acceptance of this article, the publisher recognizes that the U.S Government retains a nonexclusive, royalty-free license to publish or reproduce the published form of this contribution, or to allow others to do so, for U.S. Govermment purposes. Los Alamos National Laboratory requests that the publisher idenkify this article as work performed under the auspices of the U.S. Department of Energy. The Los Alamos National Laboratory strongly supports academic freedom and a researcher's right to publish; as an institution, however, the Laboratory does not endorse the viewpoint of a publication or guarantee its technical correctness. 


\section{DISCLAIMER}

This report was prepared as an account of work sponsored by an agency of the United States Government. Neither the United States Government nor any agency thereof, nor any of their employees, makes any warranty, express or implied, or assumes any legal liability or responsibility for the accuracy, completeness, or usefulness of any information, apparatus, product, or process disclosed, or represents that its use would not infringe privately owned rights. Reference herein to any specific commercial product, process, or service by trade name, trademark, manufacturer, or otherwise does not necessarily constitute or imply its endorsement, recommendation, or favoring by the United States Government or any agency thereof. The views and opinions of authors expressed herein do not necessarily state or reflect those of the United States Government or any agency thereof. 


\section{DISCLAIMER}

Portions of this document may be illegible in electronic image products. Images are produced from the best available original document. 


\title{
DESCRIPTION OF THE BDD-IIR: ELECTRON AND PROTON SENSORS ON THE GPS
}

\author{
by \\ T. E. Cayton, D. M. Drake, K. M. Spencer, \\ M. Herrin, T. J. Wehner, and R. C. Reedy \\ Groups NIS-2 and NIS-4, Los Alamos National Laboratory
}

\begin{abstract}
The Burst Detector Dosimeter (Block) IIR (BDD-IIR) is a multipurpose silicon detector system that is scheduled to fly on two of the first 12 spacecraft of the Global Positioning System (GPS) Block II Replenishment series as an alternative to the Burst Detector X-ray (BDX) instrument. This instrument measures energetic-particle fluxes impinging on the GPS space vehicle (SV), primarily energetic electrons trapped in the Earth's radiation belt, but also solar energetic particles and galactic cosmic rays. Absorbers located in front of eight separate silicon sensors determine energy thresholds for measuring incident particle fluxes, and the magnitude of energy loss in each sensor provides an imperfect but very good separation between ions and electrons over a wide range of energies. For each of two sensors, a conical collimator with a very small opening is used for low-energy particles. For four sensors. five small holes in a thick shield limits the flux on each sensor to manageable levels. For two sensors. solid domes are used to measure high-energy electrons and protons. These eight sensors provide eight channels that determine the electron energy spectrum from $i 7 \mathrm{keV}$ to $>5 \mathrm{MeV}$ and eight channels determine the proton spectrum from 1.3 to $>54 \mathrm{MeV}$. The radiation dose rate and total dose for a wide range of equivalent shielding thicknesses is inferred directly from the measured electron energy spectrum. Accumulations times are usually $240 \mathrm{~s}$ but can also be 24,120 , or $4608 \mathrm{~s}$. This report describes the BDD-IIR's important mechanical and electronic features. its system tests and calibrations, the commands that can be sent to it. and the data that it returns.
\end{abstract}




\section{OVERVIEW}

Instrumentation flown on the Global Positioning System (GPS) series of satellites, as part of the United States NUDET Detection System (NDS), provides global monitoring for Nuclear Detonations (NUDETs) [Higbie and Blocker, 1994]. Each of the current GPS spacecraft is configured with either a Burst Detector X-Ray (BDX) or Burst Detector Dosimeter (BDD) instrument as part of the Global Burst Detector (GBD) payload.

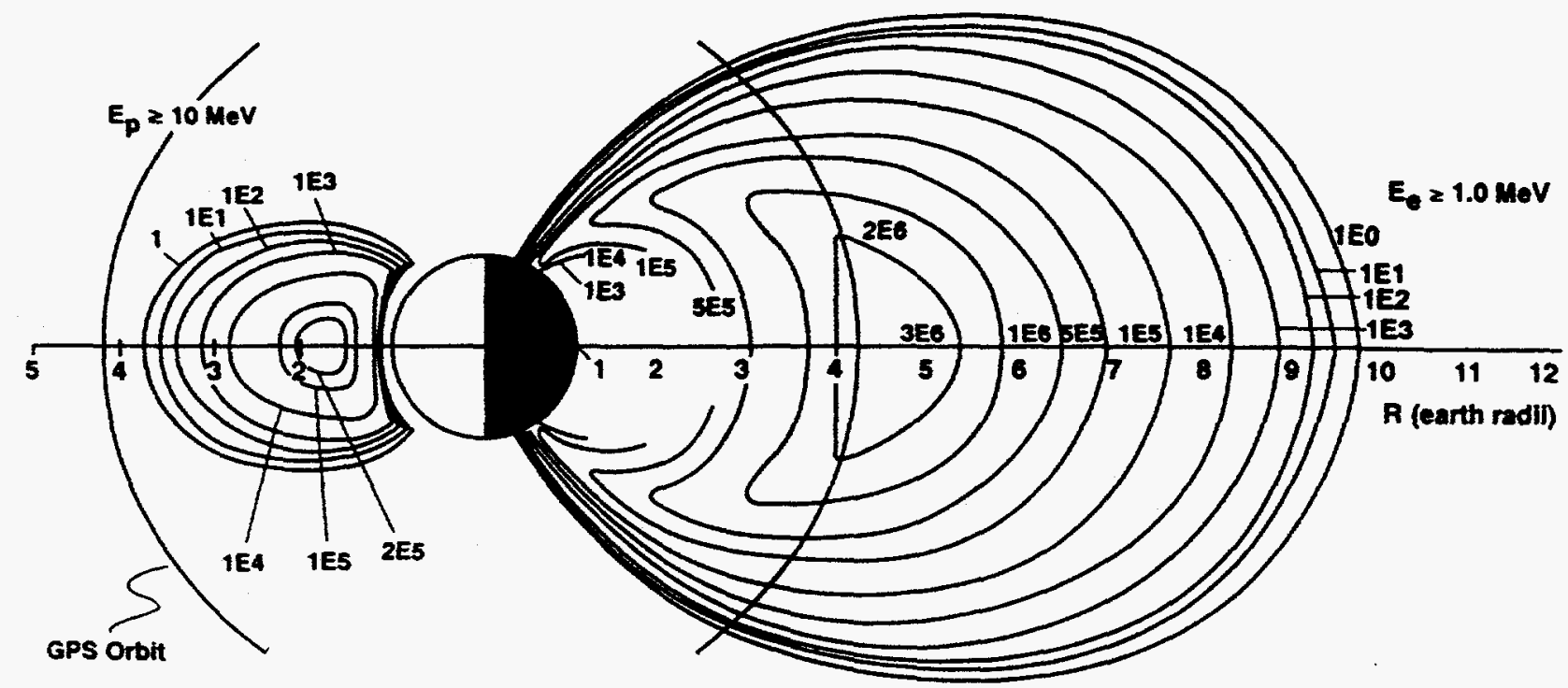

Fig. 1. A contour plot of the trapped fluxes of protons (left, the AP8MIN model) and electrons (right, the AE4 model for 1967) in the radiation belts with the GPS Block II orbit superimposed.

The GPS's 12-hour orbit, $\approx 20,000 \mathrm{~km}$ above the Earth's surface (about 4.1 Earth radii), takes each space vehicle (SV) through or near the peak-intensity region of energetic electrons trapped by the Earth's magnetic field - the outer Van Allen radiation belt four times each day. The Earth's trapped radiation, with the GPS orbit superimposed, is shown in FIGURE 1 as based on the AE4 model for electrons [Singley and Vette. 1972] and the AESMIN model for protons [Sawyer and Vette. 1976]. This is an extremely harsh and dynamic environment. These trapped energetic electrons together with solar energetic particles (which are usually encountered near the poles in the GPS's $55^{\circ}$ inclination orbit) and galactic cosmic rays (a low flux at all parts of the GPS orbit) contribute to the background counting rates in on-board instruments and cause operational anomalies and false signals. Sensitive on-board components are also aged and damaged by the primary radiation from impinging particles and by the secondary photons and particles they produce by interacting with spacecraft materials. Energetic protons degrade solar power panels and cause upsets and latchups in sensitive components.

For the 21 spacecraft GPS Block IIR, two of the first $12 \mathrm{SV}$ are scheduled to carry an energetic-particle detector, the BDD-IIR, instead of the BDX-IIR X-ray sensor, to continuously measure the background radiation environment due to magnetically-trapped 
energetic particles. solar energetic particles, and cosmic rays. The last nine Block IIR SVis will carry neither a BDX-IIR nor a BDD-IIR but. instead. a CXD instrument that combines and extends the capabilities of both the BDX-IIR and BDD-IIR.

The environmental information obtained from the BDD (or CXD) is important to assure the proper interpretation of data from the BDX X-ray detectors and other sensors, to identify the characteristic energy spectrum produced by beta decay of the radioactive fission fragments in bomb debris, and to provide, in situ, monitoring of the radiation dose delivered to spacecraft components. The data from the BDD-IIR are also valuable for operational and scientific purposes. Long-duration measurements of the space environment from GPS SVs enables understanding of the "weather" and "climate" of outer magnetospheric phenomena that affect many of the nation's space-based assets. The data are used by the Air Force's 50th Space Weather Squadron for space environmental monitoring.

The BDD-IIR's interface to the spacecraft is identical to that of the BDX-IIR. The essential difference is that the BDD-IIR continuously writes its data into the memory of the Burst Detector Processor (BDP) on the SV, which is readout and downlinked one or more times each day during routine SV contacts. The BDD gets power $(+28$ volts-DC), times, and commands from the BDP.

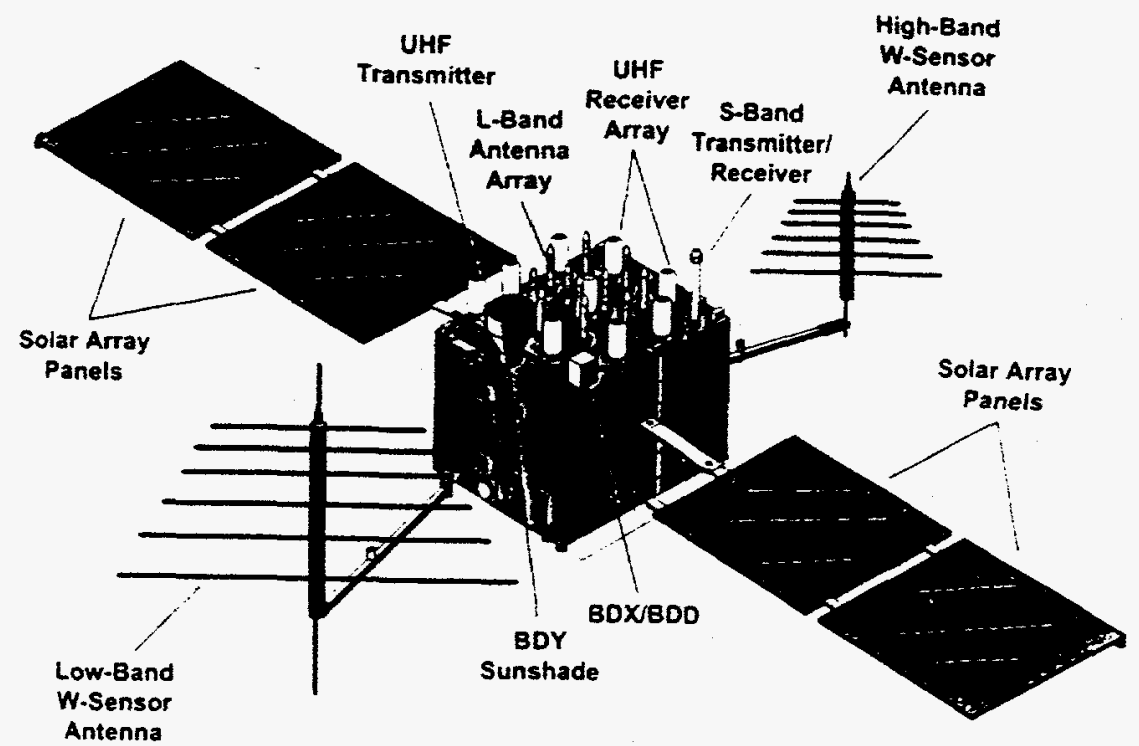

Fig. 2. Drawing of the GPS Block IIR spacecraft showing the main features and the locations of the BDD and other GBD instruments.

The BDD-IIR is mounted near a corner of the Earth-facing panel of the GPS spacecraft (FIGLRE 2). The BDD-IIR is designed to accept particles coming within $55^{\circ}$ of the instrument's axis. which is the SV's $+Z$ (Earth-facing) axis. About $1.5 \%$ of this $110^{\circ}$ field of view is obstructed by attennas and other structures on the SV.

The BDD-IIR energetic-particle instrument shown in FIGURE 3 combines some of the design features of the instruments developed for both Block I. the BDD-I SVs [Argo et al., 1980; Higbie et al., 1982; Cayton et al., 1992: and Drake et al., 1993], and Block II/IIA SVs, the BDD-II [Feldman et al., 1985]. Mechanically, the BDD-IIR somewhat 


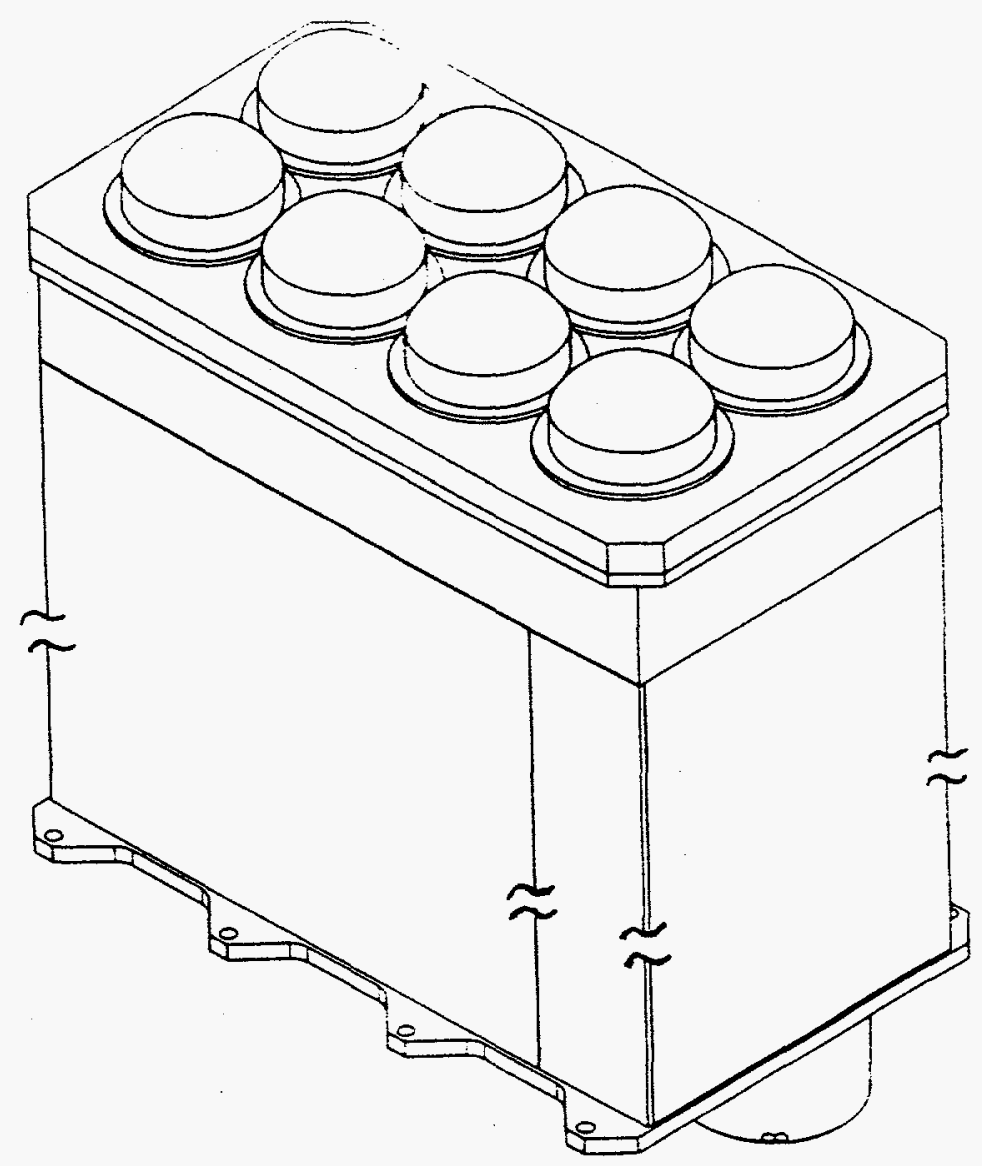

Fig. 3. Schematic of the BDD-IIR box with the eight sensors on the top. Details of the box and the tops of the sensors are not shown.

resembles the Block I instrument, but its electronics is more like that used in the Block II/IIA design. The main part of the box (not including the top plate with the eight sensors) is 11.0 inches tall, 8.5 inches wide. and 4.5 inches deep. The BDD-IIR weighs 17.2 pounds.

The BDD-IIR features eight individual channels of a "shield/filter/sensor" design that permits the detector to sample roughly half the celestial sphere (and thus to measure the omnidirectional flux) while at the same time shielding the silicon sensor elements from most of the incident particle flux. This design is a variant of the "filter/sensor" technique used in the X-ray channels of the BDX and CXD instruments. There are three types of shield/filters used on the BDD-IIR. The energy range covered by each of the eight channel is determined by the design of the shield, filter. and sensor. The information gained from the eight energy channels determines the electron energy spectrum from $7 T \mathrm{keV}$ to $>5 \mathrm{MeV}$ and also enables evaluation of the radiation dose rate and total dose for a wide range of equivalent radiation shielding thicknesses. BDD-IIR also responds to energetic protons from $1.3 \mathrm{MeV}$ to $>54 \mathrm{MeV}$. 

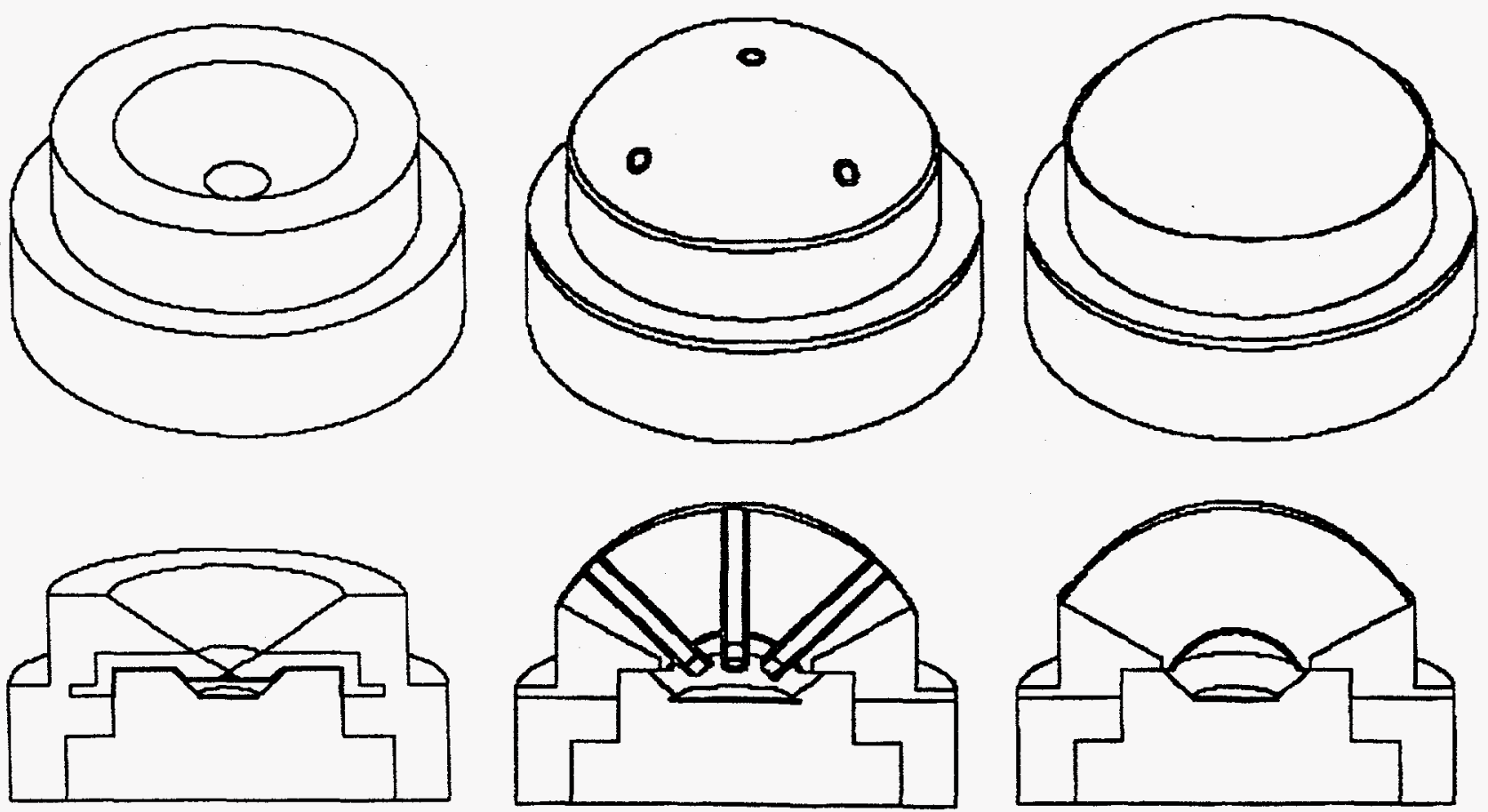

Fig. 4. Schematics of the BDD-IIR sensors, both from about $45^{\circ}$ above and a cut-away. On the left is sensor 1 or 2 with a conical collimator under a thin light-tight window (not shown) with filters at the tip above an ion-implanted $\mathrm{Si}$ detector. In the middle is sensor 4 ( similar to sensors 3.5 , and 6 ) with a "saltshaker" shield having 5 collimators with a filter at the lower end above an ion-implanted $\mathrm{Si}$ detector. On the right is sensor 7 or 8 with a solid hemispherical dome above an ion-implanted Si detector.

Detector geometries are sketched in FIGLRE 4. A 300- $\mu \mathrm{m}$-thick, $50-\mathrm{mm}^{2}$-area. ionimplanted silicon sensor in each of the eight channels is protected from the full radiation intensity by either a thick hemispherical dome (viz., right), a "saltshaker" shield (viz., middle), or a conical collimator (viz., left). For the four saltshaker shields. particles are admitted to the sensor through five cylindrical, radially oriented, gold collimators that pierce a thick beryllium and gold dome shield. one at $0^{\circ}$ and four equally-spaced at $45^{\circ}$. Beryllium filters at the bottoms of the collimating apertures determine the energy range covered by each channel. The diameter of the collimating apertures in each shield was matched to the thickness of the filter (and. therefore, the threshold energy of that channel) so that the expected counting rates in all channels would be comparable.

One of the two solid dome shields is identical to the beryllium and gold shields used for the four saltshakers except that it has no collimators: this channel provides a measurement of the contribution that $z 6-\mathrm{MeV}$ electrons and $>54 \mathrm{MeV}$ protons make to the four mediumenergy-range channels by penetrating their shields. The second dome shield lacks the inner 
layer of gold and is penetrated by electrons with energies $24 \mathrm{MeV}$ and by protons with energies $>50 \mathrm{MeV}$.

The two conical collimators are identical but have different filters behind the exit aperture to determine the energy ranges covered by each channel. These BDX-type collimators have a $110^{\circ}$ field of view and a $0.0305-\mathrm{cm}$ diameter tungsten aperture to reduce the very large flux of electrons $>77 \mathrm{keV}$ and $>210 \mathrm{keV}$ to an acceptable level. A light-tight window of aluminized Mylar (1200 $\AA$ of aluminum deposited on each side of $1.52 \mu \mathrm{m}$-thick Mylar) covers the opening aperture of the conical collimator. The filter for the $>77 \mathrm{keV}$ channel is the same aluminized Mylar used for the light-tight windows. The $>210 \mathrm{keV}$ channel uses 0.0254-cm-thick beryllium for a filter.

Each of the dome or saltshaker shields is a thick, inhomogeneous filter consisting of a thick outer shell of beryllium and a thin inner shell of gold (replaced at low angles to the spacecraft by titanium). All of the outer beryllium hemispherical shells have the same outer and inner radii, $2.3393 \mathrm{~cm}$ and $0.8585 \mathrm{~cm}$, respectively. The inner radius of the gold shell is $0.8179 \mathrm{~cm}$.

Table I. Physical Characteristics of the 8 BDD-IIR Channels

\begin{tabular}{|c|c|c|c|c|c|}
\hline $\begin{array}{l}\text { Channel } \\
\text { Number }\end{array}$ & $\begin{array}{c}\text { Filter } \\
\text { Material }\end{array}$ & $\begin{array}{c}\text { Filter } \\
\text { Thickness } \\
(\mathrm{mm})\end{array}$ & $\begin{array}{l}\text { Number } \\
\text { of Holes }\end{array}$ & $\begin{array}{c}\text { Hole } \\
\text { Diameter } \\
(\mathrm{mm})\end{array}$ & $\begin{array}{c}\text { Geometric } \\
\text { Factor } \\
\left(\mathrm{cm}^{2} \mathrm{sr}\right)\end{array}$ \\
\hline 1 & Mylar/Al & $0.00152^{a}$ & $1^{b}$ & 0.305 & $6.21 \times 10^{-4}$ \\
\hline 2 & $\mathrm{Be}$ & 0.254 & $1^{b}$ & 0.305 & $6.21 \times 10^{-4}$ \\
\hline 3 & $\mathrm{Be}$ & 0.737 & 5 & 1.500 & $4.64 \times 10^{-4}$ \\
\hline 4 & $\mathrm{Be}$ & 1.829 & 5 & 1.900 & $1.19 \times 10^{-3}$ \\
\hline 5 & $\mathrm{Be}$ & 3.962 & j & 2.400 & $3.03 \times 10^{-3}$ \\
\hline 6 & $\mathrm{Be}$ & 7.874 & 5 & 4.415 & $4.99 \times 10^{-2}$ \\
\hline 7 & $\mathrm{Be}$ & $14.808^{\circ}$ & 0 & - & 1.34 \\
\hline 8 & $\begin{array}{c}\mathrm{Be} \\
+\mathrm{Au}\end{array}$ & $\begin{array}{l}14.808^{c} \\
+0.406^{c}\end{array}$ & 0 & - & 1.34 \\
\hline $\begin{array}{l}{ }^{a} \text { 1.52- } \mu \\
{ }^{b} \text { Conica } \\
{ }^{c} \text { Shield }\end{array}$ & $\begin{array}{l}\text { Mylar with } \\
\text { ollimator. } \\
\text { ly. no holes }\end{array}$ & $\begin{array}{l}\text { h sides alun } \\
\text { A filters use }\end{array}$ & zed to 120 & & \\
\hline
\end{tabular}


Each saltshaker shield was drilled through with a set of five identical, radially-oriented holes that were fitted with $0.05-\mathrm{cm}$-thick cylindrical collimating tubes of gold. The diameter of the collimating apertures in each shield was matched to the threshold energy of that channel so that the expected counting rates in all channels would be comparable. Channels 3,4 , and 5 use reentrant collimators that are $1.83 \mathrm{~cm}$ long; Channel 6 uses $1.52-\mathrm{cm}$-long collimating tubes. The hole diameters plus the channel's geometric gathering power as determined by the collimators are listed in TABLE I.

Table II. BDD-IIR Penetration Energies (MeV)

\begin{tabular}{ccccccccc}
\hline \multirow{2}{*}{ Channel } & \multicolumn{3}{c}{ Electrons $^{a}$} & & \multicolumn{3}{c}{ Protons $^{b}$} \\
\cline { 2 - 4 } \cline { 6 - 8 } & $12 \%$ & $50 \%$ & $88 \%$ & & $12 \%$ & $50 \%$ & $88 \%$ \\
\hline 1 & 0.08 & 0.08 & 0.13 & & 1.27 & 1.27 & 1.27 \\
2 & 0.21 & 0.25 & 0.36 & & 5.27 & 5.32 & 5.37 \\
3 & 0.40 & 0.48 & 0.66 & & 9.37 & 9.46 & 9.55 \\
5 & 0.76 & 0.90 & 1.21 & & 15.6 & 15.8 & 16.0 \\
6 & 1.39 & 1.64 & 2.16 & & 24.1 & 24.3 & 24.5 \\
7 & 2.47 & 2.88 & 3.69 & & 35.2 & 35.5 & 35.8 \\
8 & 4.26 & 4.97 & 6.18 & & 50.0 & 50.4 & 50.9 \\
& 5.67 & 7.57 & 10.9 & & 53.7 & 54.1 & 54.6 \\
\hline
\end{tabular}

${ }^{a}$ Energy for which the given percentage of incident electrons emerge from filter with $>0.074 \mathrm{MeV}$.

${ }^{b}$ Energy for which the given percentage of incident protons emerge from filter with $>1.16 \mathrm{MeV}$.

Beryllium filters that determine the incident-energy threshold of the channel for electrons and protons are inserted into the exit aperture of each collimator. The thicknesses of the filters are given in TABLE I. The energy characteristics of the eight BDD-IIR channels, listed in TABLE II, are derived from the mechanical characteristics given in TABLE I and the deposited-energy threshold of the silicon sensor elements. Electrons scatter more while passing through matter, hence the greater range of penetration energies than for protons. 


\section{CIRCUIT DESCRIPTION}

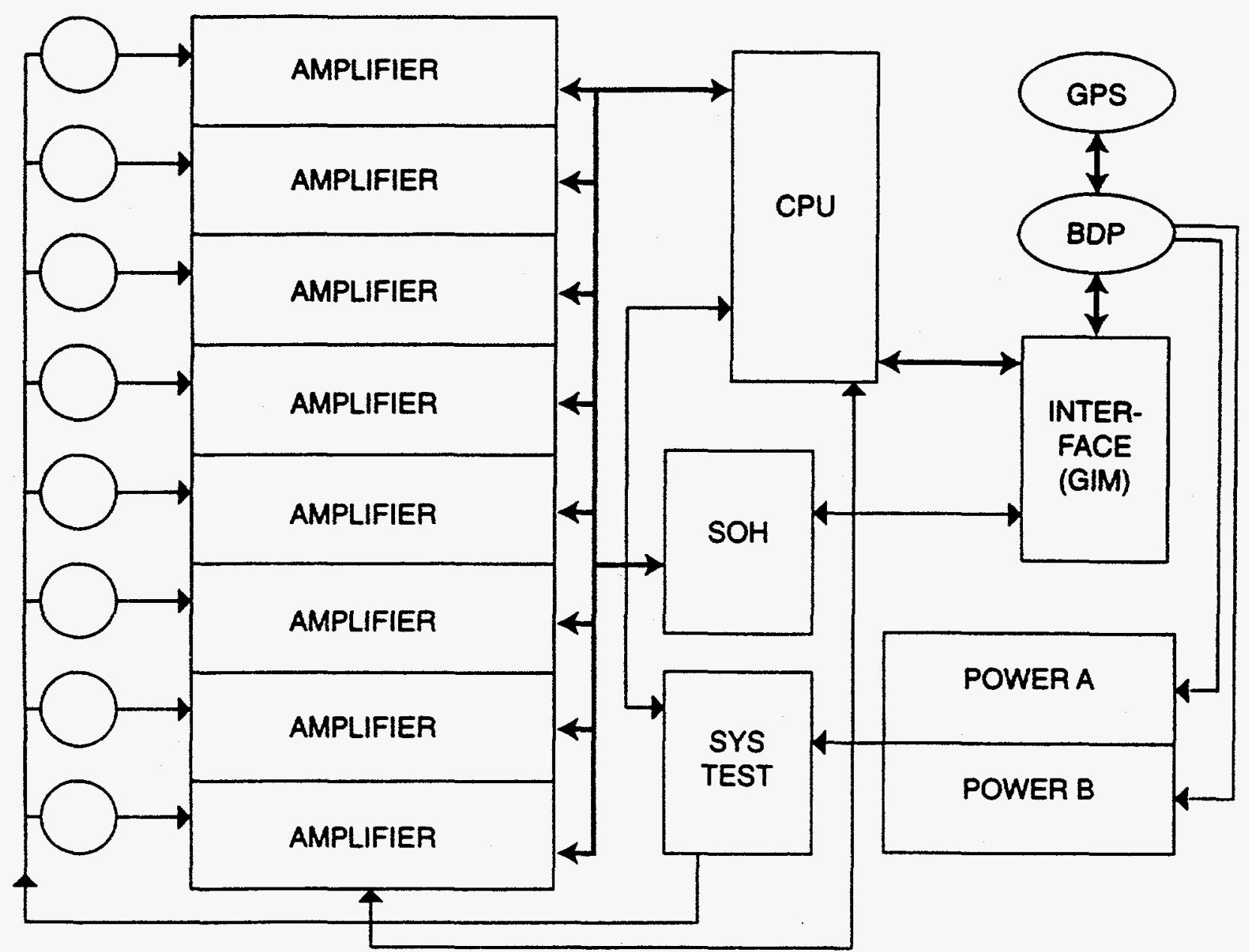

Fig. 5. Simplified block diagram of the BDD-IIR electronics showing the system test module (SYS TEST) and leads, the amplifiers for the 8 sensors, the 2 redundant power supplies, the Central Processing Unit (CPU), State-of-Health ( $\mathrm{SOH}$ ) module, the main data links (heavy lines), and the interface to the BDP and GPS spacecraft.

Although the BDD-IIR somewhat resembles the Block I instrument mechanically, BDD-IIR's particle counting electronics is much simpler than the dose accumulators used for BDD-I [e.g., Cayton et al., 1992]. A simplified block diagram of BDD-IIR is shown in FIGURE 5 . Each of the eight channels consists of an ion-implanted silicon detector and associated analog electronics and data handling logic. FIGURE 6 is a block diagram of one of the eight nominally identical circuits, each of which splits into two channels: a lowerdeposited-energy channel that responds mainly to electrons and a higher-deposited-energy channel that responds mainly to protons. 


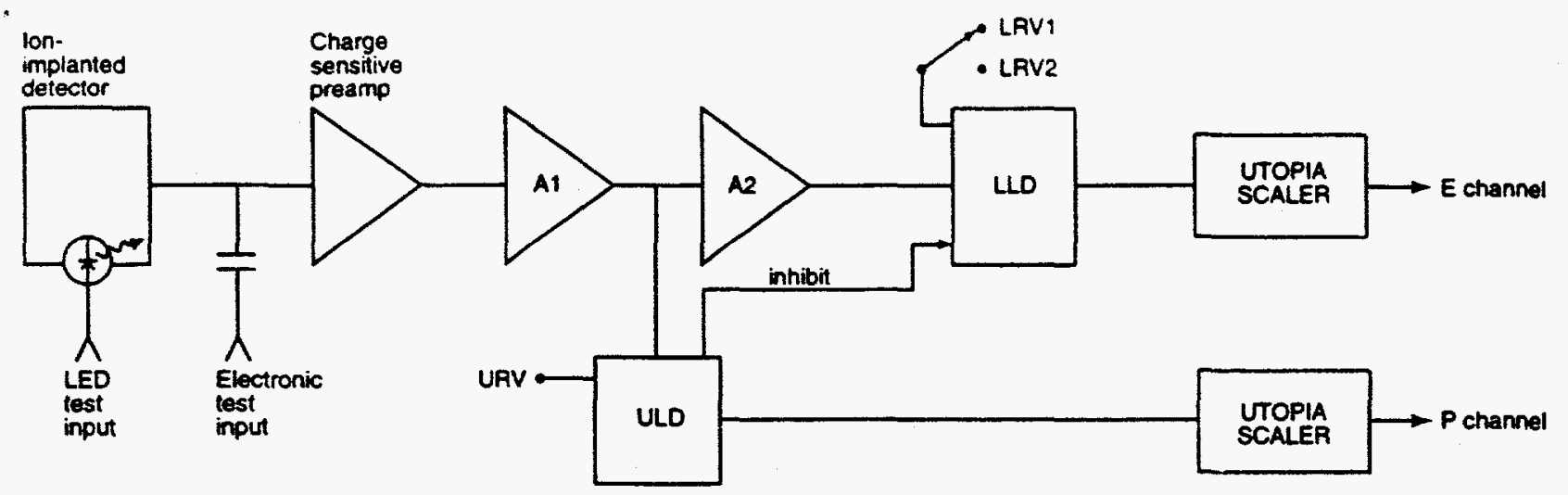

Fig. 6. The electronic circuit used for each of the 8 sensors that generate a proton (P) and an electron (E) channel. A1 and A2 are amplifiers, URV is the Upper Reference Voltage for the Upper Level Discriminator (ULD) and LRV1 and 2 are the Lower Reference Voltages for the Lower Level Discriminator (LLD).

When a charged particle deposits energy in the active volume of the ion-implanted detector, a charge-pulse (proportional to the deposited energy) is amplified by the chargesensitive preamplifier, the output of which is a shaped pulse with amplitude proportional to the energy deposited in the detector. This pulse is amplified and pulse-height analyzed by two level discriminators. If the pulse amplitude falls below the lower level discriminator (LLD) threshold, set by the lower reference voltage (LRV), nothing further happens. The LLD threshold is selected by a command from two possible values: LRV1 (Level 1) or LRV2 (Level 2). Under normal circumstances, Level 1 is selected. However, if a channel becomes noisy, selecting Level 2 raises the LLD threshold for all eight E-channels to reduce the counting rates.

If the pulse amplitude exceeds the LLD threshold but not the upper level discriminator (ULD) threshold, set by the upper reference voltage (URV), the "E" utopia scaler increments by one count. If the pulse amplitude exceeds the ULD threshold, the "P" utopia scaler increments by one count (the LLD is inhibited). Thus charged particles score in only one of two ways: (1) the E-scaler increments by one when the pulse amplitude falls between the LLD and ULD thresholds, or (2) the P-scaler increments by one when the pulse amplitude falls above the ULD threshold. If two or more particles that each generate sub-threshold pulses occur within about $2 \mu$ s of each other, their pulses could "pile up," summing to produce a pulse that exceeds the threshold and thus increments a scaler.

The BDD-IIR instrument includes redundant power supplies, unlike previous BDD versions. Instrument power is commanded on or off and selection of either the A-side or B-side power supply are accomplished through bits D1 and D0, respectively, of serial magnitude command (SMC) 43. Bit D1 is 0 for the $A$ side and 1 for the B side. Bit D0 is 1 for BDD power on and 0 for power off. The two power supplies are near the bottom of the box.

Right above the power supplies are four boards with the electronics: the interface, the CPU, the state-of-health, and the system-test boards. Near the top of the box are two amplifier boards and a bias board. All of these boards are connected to the mother board. 


\section{SYSTEM TESTS}

The BDD-IIR instrument, unlike previous versions of the BDD, includes electronic system test functions that allow stimulation of the electronics in two ways, either by injecting a charge or using light emitting diodes (LEDs). These system tests use the two test inputs shown in Fig. 6. A charge can be injected via the electronic test input capacitor into the charge-sensitive preamplifiers, or the detectors can be excited with LEDs for an "end-to-end" test of each channel. In either case, the system test module, denoted "TEST" in Fig. 5, sends 1875 electronic pulses into each channel (1250 Hz for 1.5 seconds into the electron channels followed by 1.5 seconds into the proton channels). Because the light output of the LEDs depends on temperature, the light pulse may not be sufficient to stimulate the correct channels at extreme instrument temperatures.

During routine operation, an electronic system test will be performed once per day. The BDD system test occurs during the test sequence for the other GBD instruments rather than independently. The BDD system test is enabled by bit D1 of SMC 28 and executed when bit 5 of SMC 28 is 1 . The system test occurs at the system test trigger, which can be commanded by SMC 28 to be the next X1 epoch (almost immediately) or when a time specified in SMC 27 occurs (which occurs within $384 \mathrm{~s}$ of receipt of the command).

The BDD-IIR L3 data frame (Table A5) includes a status word (byte 40) with a bit that indicates whether or not a system test occurred during the collection interval. Another bit in the status word indicates whether that test used electronic pulses or LEDs. System tests are planned for routine operation. Except for the least-significant bit, the BDD status word reflects the information and meaning of SMC 39, shown in Table A1. The least-significant bit of the BDD status word indicates whether or not a system test occurred during that accumulation interval. Accumulations that include a system test must be corrected by subtracting 1875 counts from the actual total (unscaled) for each channel. A value of one in the least-significant bit of the BDD status word signals the need for these corrections.

\section{CALIBRATION}

Absolute calibration of the deposited-energy thresholds was accomplished through use of radioactive sources and a precision pulser as follows. First, with the shields removed and a radioactive source placed directly over one of BDD-IIR's sensors, the positions of the $59.5-\mathrm{keV}$ americium-241 gamma ray and the 481-, 561-, 975-, and 1047-keV bismuth-207 electron lines were recorded in a multichannel analyzer (MCA) attached to the output of BDD-IIR's first amplifier stage. The radioactive source was then removed. Next, the combination of the precision pulser and the injection capacitor for the channel was calibrated by matching each source feature recorded by the MCA. The MCA was then removed, and the thresholds associated with the channel were carefully mapped with the calibrated pulser. The threshold maps were least-squares fitted to determine a central energy and an equivalent full-width-at-half-maximum (FWHM) parameter for each threshold. (For an 
Table III. Average Thresholds for BDD-IIR Level Discriminators

\begin{tabular}{cccc}
\hline & \multicolumn{2}{c}{ LLD } & ULD \\
\cline { 2 - 3 } & Level 1 & Level 2 & \\
\hline $\begin{array}{c}\text { Deposited Energy } \\
\text { Threshold (keV) }\end{array}$ & $74.3 \pm 2.5$ & $93.3 \pm 2.9$ & $1161 \pm 28$ \\
$\begin{array}{c}\text { Threshold Temp. } \\
\left.\text { Coeff. (keV } /{ }^{\circ} \mathrm{C}\right)\end{array}$ & $0.061 \pm 0.011$ & $0.082 \pm 0.015$ & $0.562 \pm 0.131$ \\
$\begin{array}{c}\text { Threshold } \\
\text { FWHM (keV) }\end{array}$ & $21.1 \pm 5.2$ & $20.9 \pm 5.2$ & \\
\hline
\end{tabular}

Values are averages for the 8 channels on the 3 BDD-IIR flight units - see text for details.

error-function-like transition, these parameters are defined in terms of the $50 \%$ point and the distance between the $12 \%$ and $88 \%$ points, respectively.)

TABLE III summarizes the measurements made on all eight channels of each of all three BDD-IIR flight units. Mean values and standard deviations are given for each set of 24 measurements. To determine dependences upon temperature, all thresholds were mapped at three operating temperatures. (The thresholds were found to exhibit only a weak dependence on temperature.) Small differences in the actual components used in the individual channels account for the small standard deviations in TABLE III.

\section{UTOPIA SCALERS}

The BDD-IIR accumulates its data in 16 utopia scalers operating in a pseudologarithmic mode with a compression factor of 256 . Each scaler generates two eight-bit bytes of output. The output data are decompressed as follows. (IFIX truncates any fraction, e.g., $N_{i}=0$ for $Z_{i}=255$ but $N_{i}=1$ for $Z_{i}=256$.)

$$
\begin{gathered}
Z_{i}=256 *(\text { high byte })_{i}+(\text { low byte })_{i}, \\
N_{i}=I F I X\left(Z_{i} / 256\right), \\
X_{\min }=Z_{i} *\left(N_{i}+1\right)-128 * N_{i} *\left(N_{i}+1\right), \\
X_{\max }=X_{\min }+N_{i}, \\
X_{\text {bar }}=Z_{i} *\left(N_{i}+1\right)-128 * N_{i} *\left(N_{i}+1\right)+N_{i} / 2,
\end{gathered}
$$

where (low byte $)_{i}$ and (high byte) $)_{i}$ are the low-order and high-order output bytes from the ith utopia scaler, $Z_{i}$ is the compressed accumulation, $X_{b a r}$ is the mean value of the 
uncompressed accumulation $\left(X_{i}\right)$, and $X_{\min }$ and $X_{\max }$ are the endpoints of the range. $N_{i}$ is the number of different input counts, $X_{i}$, that yields the same output count, $Z_{i}$.

\section{BDD-IIR MICROPROCESSOR}

An internal microprocessor controls the BDD-IIR. Spacecraft timing signals (X1 EPOCH) constitute the BDD-IIR's 1.5-s heartbeat. Upon receiving a selected number of EPOCHs (normally 160, but 16, 80, or $\mathbf{3 0 7 2}$ may be selected, with the corresponding accumulation time interval normally $240 \mathrm{~s}$ but could be $24 \mathrm{~s}, 120 \mathrm{~s}$, or $4608 \mathrm{~s}$ ), the microprocessor reads and formats the 32 data bytes plus 4 status bytes $(+250 \mathrm{~V}$ level, $+5 \mathrm{~V}$ level, sensor-deck temperature, and status) into a BDD data subframe. Upon completion of a data frame ( 1 or 7 subframes), the microprocessor sends the frame to the Burst Detector Processor (BDP) where it is written into the BDP memory for subsequent downloading during routine contact with the SV one or more times each day.

There are 16,384 8-bit bytes of memory in the BDP dedicated to BDD-IIR data, which translates into about 30 hours of operation with the 240-s accumulation interval.

\section{NUMERICAL SIMULATIONS}

The responses of the sixteen channels (one electron channel and one proton channel for each of the eight sensors) are being computed numerically with the Monte Carlo codes LAHET (Los Alamos High Energy Transport) [Prael and Lichtenstein, 1989] and MCNP (Monte Carlo N-Particle) [Briesmeister, 1993]. LAHET is a transport code that computes the motion of the primary protons and of particles made by interactions; MCNP follows the primary electrons and their issues. Both of these codes permit multiple materials and complicated geometrical objects. These state-of-the-art transport codes have been developed by the Radiation Transport Group at Los Alamos over many years. Similar calculations were done for the BDD-I [Cayton et al., 1992] and BDD-II.

The standard codes were modified to allow pseudo-pulse-height analysis of energy deposited in the cells representing the active volume of the silicon surface-barrier detectors. The desired result of a Monte Carlo simulation is a pulse-height distribution of energy deposited by all primary and secondary particles and photons. Channel responses are derived from these pulse-height distributions.

\section{BDD COMMANDS}

Serial magnitude commands ( $S M C s$ ) $38,39,3 A$, and $3 B$ (numbered in hexadecimal) can be sent to the BDD (or BDX). These commands are stored in the BDP upon receipt. They are sent to the BDX/BDD if it is enabled. Upon receipt of the commands, the BDX/BDD stores them and performs the required actions. SMC 38 is only for the BDX (and is not given here). SMC 39 is only for the BDD. SMC 3A and 3B apply to both the BDD and BDX. Details on these commands are given in Appendix 1. The important parameters in these commands are summarized below. 
SMC 39 controls the BDD particle data parameters. The BDD can be set to accumulate particle data for $24,120,240$, or 4608 seconds. The $24-$ and 4608 -second periods are normally not used in orbit but only for ground testing. At the end of each collection interval, the instrument completes a single subframe of the BDD data frame, 32 data bytes and $4 \mathrm{SOH}$ bytes. The BDD can be set to generate either 1 or $i$ subframes in each data frame. The lower threshold (deposited energy) for the eight sensors, can be set as either low $(74 \mathrm{keV})$ or high $(93 \mathrm{keV})$. The system test can be selected to be either electronic or with the light emitting diodes (LEDs). It is possible to disable the collection of BDD data frames.

SMC 3A is used to control the transfer of BDD messages to the BDP. This command can be generated internally by the BDP if enabled. The BDD can be told not to send any $\mathrm{BDD}$ collections until requested. Another bit tells the BDD to ignore any memory readout commands from the BDP until this bit is reset. The BDD can be instructed not to transfer any more SOH or status messages until told to do so.

SMC 3B is used to force a Global Interface Module (GIM) reset, select BDD GIM parity options, enable/disable BDD message receive error checking, and to force a BDD $\mathrm{SOH}$ transfer. This command will be generated internally by the BDP in case of certain problems.

\section{OTHER COMMANDS AFFECTING THE BDD}

Besides the BDD commands (SMCs 39, 3A, and 3B), other commands can affect the BDD and its data. Some commands are generated by the BDP and are not considered here. Some of the more important ones that are sent from the ground are given here. Other commands can affect what is sent from the BDD to the ground by the BDP, such as $\mathrm{SOH}$ and data frames, but are not discussed here.

Several community commands can affect the BDD, such as system tests. Bit D1 of SMC 28 set to 1 enables the BDD system test. Bit D5 of SMC 28 set to 1 executes a system test. Other commands discussed in Section 7.3.5 determine the nature of the system-test trigger (on the next X1 Epoch, on a ZTIME match with a time in S.MC 27, or a delayed system test with SMC 4B). S.IC $2 \mathrm{C}$ can be used to clear and test the BDD's memory: but such memory tests and clears are normally not done because they destroy the memory's contents. SMC 33 controls the transfer of a memory read out from the BDD for an S-band dump.

Several commands to the BDP affect the BDD. Bit D5 of SMC 41 set to 1 initializes the BDD's processor. Bit D6 of SMC 42 set to 1 disables the BDD's functions. Bit D0 of SMC 43 turns the BDD power on if 1 and off if 0 . Bit D1 of SMC 43 selects BDD power from $A$ if 0 and from $B$ if 1 .

SMC 45 can affect the collection of BDD data by the BDP. If bit D3 of SMC 45 is set to 1, the BDP will not overwrite the BDD section of the BDP MP's memory with event-initiated L3 data. However, if this bit is set to 0, the BDP can overwrite this memory. Usually $\mathrm{L} 3$ events will not overwrite the BDD section of memory, but an overwrite is possible for high event data rates into the BDP. When this BDD memory section is overwritten by L3 event data, the MP issues a SMC 39 with bit D4 = 1 to the BDD to 
disable event collection. A SMC 39 from the ground with $\mathrm{D} 4=0$ is required to re-enable collection of BDD data by the BDP. This commands has to be sent after the MP has terminated the L3 overwrite mode.

\section{BDD MESSAGE FORMATS}

The BDD has five types of messages that it sends serially to the BDP. Four are briefly described below with details in Appendix 2: status messages, state-of-health messages. data collections, and memory readouts. The fifth message type consists of EOM byte pairs sent in initialization and GIM resets.

The BDD has two status messages. One confirms a recent initialization. The other tells the BDP that the BDD cannot sync up on the ender message bytes and that the BDP should reset the GIM connected to the BDD serial link.

The BDD provides state-of-health $(\mathrm{SOH})$ messages to the BDP every six seconds. There are two types of $\mathrm{SOH}$ messages, regular and extended. The regular $\mathrm{SOH}$ message contains a header byte, two Z-time bytes, 48 data bytes, and the two EOM bytes. The extended SOH messages included additional bytes for a total of 125 data bytes.

The BDD has a total of sixteen channels that are split into eight electron and eight proton channels. Each channel has two bytes for each collection subframe. There can be either 1 or 7 subframes per collection message. Also sent with each subframe are four additional bytes - the high-voltage $(+250 \mathrm{~V})$ monitor, the low-voltage $(+5 \mathrm{~V})$ monitor, the temperature of the sensor deck, and the BDD status word. BDD data frames are normally transmitted on S-band but can be sent via the L3 downlink as non-event data.

The BDD can also dump the contents of its memory to the BDP upon command.

\section{ACKNOWLEDGMENTS}

Nany personnel at Los Alamos helped in designing. building, and testing the BDDIIR. including R. D. Dingler (flight software). P. R. Ma jerus (ground support equipment i. J. C. Ingraham (calibration), J. Masarik (numerical simulations), and R. D. Belian (sensors). Some of the information on commands and data formats were provided by our colleagues at the Sandia National Laboratories, Albuquerque, who integrated the BDD into the GBD package. All the work reported herein was supported by and performed under the auspices of the United States Department of Energy. 


\section{REFERENCES}

H. V. Argo, D. N. Baker, R. D. Belian, L. K. Cope, and P. R. Higbie (1980), "The BDD: A Dosimeter for the Global Positioning System," Los Alamos Scientific Laboratory report $L A-8421-M S, 9$ pp., (Oct. 1980).

J. F. Briesmeister (1993), "MCNP - A General Monte Carlo N-Particle Code," Los Alamos National Laboratory report LA-12625-M (Nov., 1993).

T. E. Cayton, P. R. Higbie, D. M. Drake, R. C. Reedy, D. K. McDaniels, R. D. Belian, S. A. Walker, L. K. Cope, E. Noveroske, and C. L. Baca (1993), "BDD-I: An Electron and Proton Dosimeter on the Global Positioning System Final Report," Los Alamos National Laboratory report $L A-12275,71$ pp., (May 1992).

D. M. Drake, T. E. Cayton, P. R. Higbie, D. K. McDaniels, R. C. Reedy, R. D. Belian, S. A. Walker, L. K. Cope, E. Noveroske, and C. L. Baca (1993), "Experimental Evaluation of the BDD-I Dosimeter for the Global Positioning System," Nucl Instrum. \& Methods Phys. Res., A333, 571-588.

W. Feldman, W. Aiello, D. Drake, and M. Herrin (1985), "The BDD II: An Improved Electron Dosimeter for the Global Positioning System," Los Alamos National Laboratory report $L A-10453-M S, 18 \mathrm{pp}$. , (July 1985).

P. R. Higbie, R. D. Belian, H. V. Argo, and D. N. Baker (1982), "Calibration of an Electron/Proton Monitor for the Earth's Radiation Belt at $4 \mathrm{R}_{E}$," Los Alamos National Laboratory report $L A-9195-M S, 26$ pp., (Mar. 1982).

P. R. Higbie and N. K. Blocker (1994), "Detecting Nuclear Detonations with GPS," GPS World, Feb. 1994.

R. E. Prael and H. Lichtenstein (1989), "User Guide to LCS: The LAHET Code System," Los Alamos National Laboratory document LA-UR-89-3014 (September 1989).

D. M. Sawyer and J. I. Vette (1976), "AP-8 Trapped Proton Environment for Solar Maximum and Solar Minimum," National Space Science Data Center report NSSDC/WDC-A-R\&S 76-06 (NASA-GSFC TMS-72605) (Dec. 1976).

G. W. Singley and J.I. Vette (1972), "The AE-4 Model of the Outer Radiation Zone Electron Environment," National Space Science Data Center report NSSDC 72-06. 


\section{APPENDIX 1. BDD-IIR COMMANDS}

Serial magnitude commands (SMCs) 38, 39, 3A, and 3B can be sent to the BDD (or the BDX). These commands are stored in the BDP upon receipt. They are sent to the $\mathrm{BDX} / \mathrm{BDD}$ if it is enabled. Upon receipt of the commands, the BDX/BDD stores them and performs the required actions. Commands sent elsewhere, such as to the BDP, that affect the BDD are not included here. Commands are numbered in hexadecimal plus have a short acronym. SMC 38 is only for the BDX (and is not given here). SMC 39 is only for the BDD. SMCs 3A and 3B apply to both the BDD and BDX.

For the commands below, there are two states. One is the Initial State, the value that the byte gets set to on initialization of the GBD. The Standard Configuration State is the value that the byte gets set to if the GBD does not receive any valid command within approximately three minutes of the completion of an initialization or if a command is sent for the GBD to go to the Standard Configuration.

\section{$S M C 99(B D D C M D)$}

SMC 39 controls the BDD particle data parameters. Bits 6 and 7 are not used and can be anything.

The BDD Collection Interval field (bits 4 and 5) sets the number of seconds for particle data accumulation, either $24,120,240$, or 4608 seconds. At the end of each collection interval, the instrument completes a single subframe of the BDD data frame, 32 data bytes and $4 \mathrm{SOH}$ bytes.

The Frame Size bit (bit 3) sets the number of subframes of particle data in each data frame. The BDD generates either 1 or 7 subframes in each data frame.

The Threshold bit (2) sets the lower threshold for the eight sensors, either high or low. Low (the bit is a 0 ) is $74 \mathrm{keV}$ deposited energy (Level 1) and high (1) is $93 \mathrm{keV}$ deposited energy (Level 2).

The BDD System Test controls whether the system test is electronic (1) or with the light emitting diodes (LEDs) (0).

The Data Collection Disable bit (0) is used to disable BDD data frames. If set, no collections are taken.

The format of SMC 39 (BDDCMD) is summarized in TABLE A1.

\section{SMC 3A (XMESSOF)}

SMC 3A (XMESSOF) controls the transfer of BDD messages to the BDP. This command can be generated internally by the BDP. Bits 3, 5, 6, and 7 are not used and can be anything.

The Disable BDD Collection Messages bit (bit 4) tells the BDD not to send any BDD collections until this bit is a zero. The BDD keeps collecting data but does not overwrite memory. In an high-event rate situation in which an $\mathrm{L} 3$ readout is active, the BDP Main Processor is allowed to overtake BDD memory to store event data. In this case, the BDP may issue a command making bit $4=1$ to stop the transfer of event data from the BDD. A command from the ground is needed to reset this bit to 0 and restate collection transfer. 


\begin{tabular}{ll}
\hline MSB 7 & $\mathrm{X}$ \\
6 & $\mathrm{X}$ \\
5 & BDD Collection Interval \\
4 & BDD Collection Interval \\
3 & Frame Size 1/7 \\
2 & Threshold High/Low \\
1 & System Test Electronic/LED \\
LSB 0 & Collect Disable
\end{tabular}

MSB is the most significant bit and LSB is the least significant bit. Command bits labeled $\mathrm{X}$, numbers 6 and 7 , are not used.

\begin{tabular}{ll}
\hline Collection Interval & $00=24$ seconds \\
& $01=120$ seconds \\
& $10=240$ seconds \\
& $11=4608$ seconds \\
& $0=7$ subframe in each BDD data frame \\
Frame Size & $1=1$ subframes in each BDD data frame \\
& $0=$ Level 1 \\
Lower Level Threshold & $1=$ Level 2 \\
Electronic/LED Test & $0=$ LED \\
& $1=$ electronic \\
Collection Disable & $0=$ Collect and pass all frames to the BDP \\
& $1=$ Do not pass data to the BDP \\
& Bit not used \\
\hline
\end{tabular}

The initial state is $0 \mathrm{~A}_{H}$ (where ${ }_{H}$ indicates the number is hexadecimal) $(00001010$ in binary), and the standard configuration is $22_{H}\left(\begin{array}{lllllll}0 & 0 & 1 & 0 & 0 & 0 & 1\end{array}\right)$.

The Disable BDD Memory Read Out (MRO) Message bit (2) tells the BDD to ignore any MRO commands from the BDP until this bit is 0 . If the BDD is currently transferring a MRO, it is finished but no more are sent.

The Disable BDD State of Health (SOH) Message bit (1) tell the BDD not to transfer any more $\mathrm{SOH}$ messages until this bit is 0 . If the $\mathrm{BDD}$ is currently transferring a $\mathrm{SOH}$ message, it is finished but no more are sent. If this bit is a 1 , then bit 0 of SMC 3B has no effect.

The Disable Status Messages bit ( 0 ) tells the BDD not to send any status messages. Status message transfer is allowed when this bit is changed to 0 .

The format of SMC 3A is summarized in TABLE A2. 


\begin{tabular}{cl} 
MSB 7 & $\mathrm{X}$ \\
6 & $\mathrm{X}$ \\
5 & $\mathrm{X}$ \\
4 & Disable BDD Collection Messages \\
3 & $\mathrm{X}$ \\
2 & Disable BDD MRO Function \\
1 & Disable BDD SOH Messages \\
LSB 0 & Disable BDD Status Messages \\
MSB is the most significant bit and LSB is the least significant bit. Command bits labeled \\
X, numbers 3, 5,6, and 7, are not used and can be anything. \\
\hline \multicolumn{2}{c}{$\begin{array}{l}1=\text { disabled } \\
0=\text { Disables }\end{array}$} \\
\end{tabular}

The initial state is $00_{H}(00000000)$, and the standard configuration is not applicable to this command.

\section{$S M C 3 B(X G I M F N C)$}

SMC 3B is used to force a Global Interface Module (GIM) reset, select BDD GIM parity options, enable/disable BDD message receive error checking, and to force a BDD SOH transfer. This command will be generated internally by the BDP where (1) the BDP has not received a valid transfer from the BDD in a predetermined amount of time and such transfers are enabled, (2) when the BDP gets lost in a transfer to the BDP and cannot find a valid set of end-of-messages bytes, or (3) when the BDD communicates to the BDP that it is not correctly receiving incoming messages.

Each command is discussed below followed by a summary. Bits 4 and 7 are not used and can be anything.

The BDD Channel GIM Reset bit (bit 6) tells the BDD to reset the GIM in the BDD and to send 16 end-of-message bytes to the BDP.

The Select BDD Channel GIM Parity Options bit (5) selects the parity option (even or none) used by the GIM during data transfers. Reset to a 0 , the BDD programs the GIM with even parity.

The Reset BDD "set" Commands bit (3) tells the BDD to clear the BDD set command bits to a zero value. The command bits reset to zero are SMC 28 bit 5, SMC 29 bit 5 , SMC $2 \mathrm{C}$ bits 7 and 6 , and bits 6,2 , and 0 (but not bit 3 ) of this command.

The Reset BDD Pointers, Counters \& Flags bit (2) tells the BDD to perform a "soft" reset. This allows events and data collections to be stored as if a sensor initialization had occurred but does not initialize command registers. (This differs from a hard initialization 


$\begin{array}{ll}\text { MSB } 7 & \text { X } \\ 6 & \text { BDD Channel GIM Reset } \\ 5 & \text { Select BDD Channel GIM Parity } \\ 4 & \text { X } \\ 3 & \text { Reset BDD "set" Commands } \\ 2 & \text { Reset BDD Pointers, Counters \& Flags } \\ 1 & \text { Enable BDD GIM Error Checking } \\ \text { LSB 0 } & \text { Send BDD SOH }\end{array}$

MSB is the most significant bit and LSB is the least significant bit. Command bits labeled $X$, numbers 4 and 7 , are not used and can be anything.

$\begin{array}{ll}\text { BDD Channel GIM Reset } & \begin{array}{l}0=\text { Normal mode } \\ 1=\text { Reset the GIM on the BDP/BDD } \\ \text { serial channel }\end{array} \\ \text { Select BDD Channel GIM Parity } & \begin{array}{l}0=\text { Even parity } \\ 1=\text { No parity }\end{array} \\ & 0=\text { Normal mode } \\ \text { Reset BDD "set" Commands } & \begin{array}{l}1=\text { Clear "set" commands to zero value } \\ 0=\text { Normal mode } \\ 1=\text { Perform "soft" BDD initialization }\end{array} \\ \text { Reset BDD Pointers, etc. } & \begin{array}{l}0 \text { Use received messages, ignore GIM } \\ \text { Enable BDD GIM Error Checking }\end{array} \\ \text { Send BDD SOH } & \begin{array}{l}1=\text { Disregard message if GIM error bit set } \\ 0=\text { Normal mode } \\ 1=\text { Send a BDD SOH message to the BDP } \\ \text { X }\end{array}\end{array}$

The initial state is $20_{H}(00100000)$, and the standard configuration is not applicable to this command.

in that command registers are left in their current states and the GIM and hardware are not reset.)

The Enable BDD GIM Error Checking bit (1) tells the BDD to ignore certain messages from the BDP if an error is found in the message (framing, overrun, or parity error). When this bit is reset to 0 , then the BDD tries to use all received messages.

The Send BDD SOH bit (0) tells the BDD to send a state-of-health ( $\mathrm{SOH}$ ) message to the BDP (unless bit 1 of SMC $3 A$ is 1 ).

The format of SMC $3 B$ is summarized in TABLE A3. 


\section{APPENDIX 2. BDD MESSAGE FORMATS}

The BDD has five types of messages that it sends serially to the BDP. Four of these are status messages, state-of-health messages, data collections, and memory readouts. Each of these four messages is identified by a byte (eight bits) as a header and includes the Z-time after the header. Bits 7 and 6 are different for these four types of messages. The fifth message type comprises EOM byte pairs send to the BDP upon initialization and GIM resets. All messages end with two bytes as an end-of-message (EOM) marker. The eight bits in these two EOM bytes are:

EOM $1 \quad 11110111$

EOM 211111110

Bit 7, the most significant bit (MSB) for a byte, is the first on the left. Bit 0 , the least significant bit (LSB), is the last one on the right.

\section{BDD Status Messages}

The BDD has three status messages. The header contains all zeros. One confirms a recent initialization (second byte $=18_{H}$ ). The second tells the BDP that the BDD cannot syncup on the ender message bytes and that the BDP should reset the Global Interface Module (GIM) connected to the BDD serial link (second byte $=42_{H}$ ). The third tells the BDP that the BDD acknowledges a GIM reset command (second byte $=24_{H}$ ).

\section{BDD State-of-Health Messages}

The BDD provides state-of-health ( $\mathrm{SOH}$ ) messages to the Interface Processor of the BDP about every six seconds. There are two types of $\mathrm{SOH}$ messages, regular and extended. Bits 5 and 4 in the header identify which type of $\mathrm{SOH}$ message follows. The regular $\mathrm{SOH}$ message contains a header byte, two Z-time bytes, 48 data bytes, and the two EOM bytes (for a total of 53 bytes). The extended $\mathrm{SOH}$ messages included an additional 77 bytes (mainly memory test error buffer bytes) for a total of 125 data bytes plus the five other header or EOM bytes. The data in the SOH messages are summarized in TABLE A4.

Table A4. Bytes in State-of-Health Messages

\begin{tabular}{ll}
\hline Byte & Content \\
\hline HEADER & $01 \mathrm{I}_{5} \mathrm{I}_{4} 1 \mathrm{Z}_{18} \mathrm{Z}_{17} \mathrm{Z}_{17}$ \\
& $\mathrm{I}_{5} \mathrm{I}_{4}=00$ indicates a regular SOH message \\
& $\mathrm{I}_{5} \mathrm{I}_{4}=11$ indicates an extended SOH message \\
ZTIME 1 and 2 & Bits 15 to 0 of the $\mathrm{Z}$-time $\left(\mathrm{Z}_{15}\right.$ to $\left.\mathrm{Z}_{0}\right)$ \\
DATA 1 to 26 & Command Registers $26_{H}$ to $3 \mathrm{~F}_{H}$ \\
DATA 27 & Serial Data Error Count \\
DATA 28 & Command Error Count \\
DATA 29 & No Operation Performed Error Count \\
DATA 30 & +5 Volt Monitor
\end{tabular}



DATA 31
DATA 32
DATA 33
DATA 34
DATA 35
DATA 36
DATA 37
DATA 38
DATA 39
DATA 40
DATA 41
DATA 42
DATA 43
DATA 44
DATA 45
DATA 46
DATA 47
DATA 48
-5 Volt Monitor
+12 Volt Monitor
-12 Volt Monitor
+250 Volt Bias Voltage
+250 Volt Monitor
Temperature 1 (SOH Electronic Card)
Temperature 2 (Sensor Deck)
Number of Messages Received
Number of Messages Sent
$5 \mathrm{~A}_{H}$
$\mathrm{A} 5_{H}$
Current Idle Loop Counter - High Byte
Current Idle Loop Counter - Low Byte
BDD Status Word (Read of Port $70_{H}$ )
Last Idle Loop Counter - High Byte
Last Idle Loop Counter - Low Byte
Software Version
PROM Checksum

For a regular SOH message, the two EOM bytes go here. For an extended SOH message, the following data bytes are included:
DATA 49
Marker Byte $\mathrm{FB}_{H}$
DATA 50
DATA 51
Value of Last Command $2 \mathrm{C}$ at Start of Memory Test
DATA 52
Low Byte of ZTIME at End of Memory Test
4 MSBs of Memory Test Counter, Incremented at End of Test;
Then 4 MSBs (19-16) of Memory Test Error Count
DATA 53
Next 8 MSBs of 20-Bit Memory Test Error Count
DATA 54
DATA 55
8 LSBs of 20-Bit Memory Test Error Count
DATA 56
8 MSBs of first RAM Test Error Address
DATA 57 to 124
8 LSBs of first Error Address (zeros if no errors)
DATA 125
0
EOM 1 and 2

MSBs (LSBs) $=$ Most (Least) Significant Bits; $\mathrm{X}_{H}$ means $\mathrm{X}$ is hexadecimal.

\section{$B D D$ Event Messages}

The BDD has a total of sixteen channels that are split into eight electron and eight proton channels. Each channel has two bytes for each collection subframe. There can be either 1 or 7 subframes per collection message. The number of subframes is indicated in the header byte and in bit 3 of BDD byte 2. Bytes 2 to 4 also include the Z-time. The BDD bytes are summarized in TABLE A5. 


\begin{tabular}{|c|c|}
\hline Byte & Content \\
\hline HEADER & $\begin{array}{l}10001 I_{2} I_{1} 1 \\
\left(I_{2} I_{1} \text { is } 11 \text { for } 7 \text { subframes }\right. \\
\text { and } 00 \text { for } 1 \text { subframe })\end{array}$ \\
\hline BDD 1 & Contains the 8 bits 11101011 \\
\hline BDD 2 & $\begin{array}{l}\text { Contains } 4 \text { zero bits, } \\
\text { then a } 0 \text { if } 7 \text { subframe or a } 1 \text { if one subframe, } \\
\text { then bits } 21 \text { to } 19 \text { of the } Z \text {-time }\end{array}$ \\
\hline BDD 3 and 4 & Contains bits 18 to 3 of the $Z$-time $\left(Z_{18}-Z_{3}\right)$ \\
\hline BDD 5 & Electron Channel One Data - High Byte \\
\hline BDD 6 & Electron Channel One Data - Low Byte \\
\hline BDD 7 to 20 & $\begin{array}{l}\text { Repeat for Electron Channels } 2 \text { to } 8 \\
\quad(2 \text { bytes per channel })\end{array}$ \\
\hline BDD 21 & Proton Channel One Data - High Byte \\
\hline BDD 22 & Proton Channel One Data - Low Byte \\
\hline BDD 23 to 36 & $\begin{array}{l}\text { Repeat for Proton Channels } 2 \text { to } 8 \\
\quad(2 \text { bytes per channel })\end{array}$ \\
\hline BDD 37 & Status Word One - High Voltage $(+250 \mathrm{~V})$ Monitor \\
\hline BDD 38 & Status Word Two - Low Voltage $(+5 \mathrm{~V})$ Monitor \\
\hline BDD 39 & Status Word Three - Temperature of Sensor Deck \\
\hline BDD 40 & $\begin{array}{l}\text { Status Word Four - BDD Status Word } \\
\text { (Bit } 0 \text { in this word is the System Test Indicator) }\end{array}$ \\
\hline
\end{tabular}

For a regular $\mathrm{SOH}$ message, the two EOM bytes go here. For an extended $\mathrm{SOH}$ message, the following data bytes are included:

If the message contains only one subframe, the two EOM bytes follow BDD byte 40 . If the message contains 7 subframes, each sequence of 36 bytes (BDD bytes 41 to 76 for subframe 2) have the same contents as bytes 5 to 40 above. Subframes 3 through 7 are in BDD bytes 77-112 through 221-256.

EOM 1 and 2 are after the last data byte (BDD byte 40 or 256 ).

\section{$B D D$ Memory Readout}

The BDD can dump the contents of its memory to the BDP upon command by command byte 33 . The $\mathrm{BDD}$ has two $16 \mathrm{~K}$ byte memory sections. A memory readout (MRO) provides $16 \mathrm{~K}$ bytes that are broken down into 64 contiguous messages with 256 data bytes each. Each MRO messages has three header bytes (that identify the message as a MRO and contain the starting address of memory) and the two EOM bytes. 